\title{
Measurement System Design Using Damage Scenarios
}

\author{
Prakash Kripakaran ${ }^{1}$, Suraj Ravindran ${ }^{2}$, Sandro Saitta ${ }^{3}$ and Ian F. C. Smith ${ }^{4}$ \\ ${ }^{1}$ IMAC, Struct. Eng. Inst., Station 18, Ecole Polytechnique Fédérale de \\ Lausanne (EPFL), CH-1015 Lausanne, Switzerland, prakash.kripakaran@epfl.ch \\ ${ }^{2}$ Struct. Eng. Inst., Station 18, Ecole Polytechnique Fédérale de Lausanne \\ (EPFL), CH-1015 Lausanne, Switzerland, suraj.ravindran@epfl.ch \\ ${ }^{3}$ School of Comp. and Comm. Sc., Station 18, Ecole Polytechnique Fédérale de \\ Lausanne (EPFL), CH-1015 Lausanne, Switzerland, sandro.saitta@epfl.ch \\ ${ }^{4}$ Struct. Eng. Inst., Station 18, Ecole Polytechnique Fédérale de Lausanne \\ (EPFL), CH-1015 Lausanne, Switzerland, ian.smith@epfl.ch
}

\begin{abstract}
This paper presents a measurement system design methodology that incorporates damage scenarios. One of the goals of a measurement system is to detect damage for safety and for management of maintenance activities. This study builds upon previous research at EPFL in the area of model based diagnosis. An existing bridge in Switzerland is used to illustrate how damage scenarios are identified and a population of models are generated. A greedy algorithm is used to place sensors such that there is maximum separation between the predictions of the models in the population.
\end{abstract}

\section{Introduction}

There are hundreds of ways to measure physical phenomena in structures and many new measurement technologies are emerging every year. However, inferring meaningful information from data remains a major difficulty. A systematic approach to interpretation of measurement data employs methodologies developed in the field of system identification (Ljung, 1999). System identification involves determining the state of a system and values of system parameters through comparisons of predictions with measurements. Once identified, models can support decision-making with respect to future maintenance and repair. Since system 
identification is an inverse problem and errors are involved in both measurement and modeling, many models may be able to explain the same measurement (Smith, 2005). Therefore it is of interest to configure measurement systems such that maximum separation between candidate models can be achieved (Saitta et al., 2006).

Sensors are extensively used in activities such as bridge diagnosis (RobertNicoud et al., 2005a), construction quality control (Akinci et al., 2006) and water network maintenance (Berry et al., 2005). Due to the lack of a systematic approach for the configuration of measurement systems, engineers currently rely on their experience and judgment to decide upon the location and the type of sensors. Computational approaches to aid engineers in configuring measurement systems have the potential to maximize the likelihood of identifying the correct model for the structure. Robert-Nicoud et al. (2005b) proposed an iterative greedy algorithm that places the next sensor at a location that gives maximum separation between predictions of candidate models. Key steps in this methodology include (1) the simulation of a set of candidate models based on modeling assumptions provided by the engineer and (2) the evaluation of the performance of a sensor when placed at a particular location. This paper describes how we build on the methodology developed by Robert-Nicoud et al (2005b) through incorporating damage scenarios.

An objective of employing sensors on infrastructure systems such as bridges is damage detection. For successful damage detection, it is essential that appropriate sensors are placed at locations such that the chances of detecting damage are maximized. Once the engineer identifies damage scenarios, these scenarios are simulated. Damage scenarios to be considered depend on factors such as the material, the structural system, boundary conditions, loads and geographical location. For instance, one of the piers in a bridge may not be functioning as a support after a flood or an earthquake. Combinations of different scenarios are also possible. Optimal sensor placement is one that gives maximum separation between predictions of the effects of damage scenarios.

This paper uses the Schwandbach bridge in Switzerland to illustrate the importance of incorporating damage scenarios into the measurement system design process. The next Section is a review of the measurement system design methodology. This is followed by a description of the bridge that is used to illustrate the strategy. Damage scenarios that are common to concrete bridges are included and this results in a set of 5000 models. The paper ends with a presentation of first results and a description of future work.

\section{Measurement System Design}

Sensor-data driven decision support systems can be the basis of proactive management of structural facilities. It has the potential to improve the performance and reduce the life-cycle cost over a broad range of infrastructure systems (Garrett et al., 2006). For effective decision support, it is essential that sensor systems are 
configured to measure responses at locations such that the measured data can be meaningfully interpreted. Robert-Nicoud et al. (2005b) developed a sensor placement methodology that consists of two key steps - (1) Generation of candidate models and (2) Sensor performance evaluation at each location.

Robert-Nicoud et al. (2005b) used the methodology shown in Figure 1 for generating the candidate models. Since generating all possible models is combinatorial, a population of models is randomly generated using assumptions made by the engineer. Each model is evaluated by finite element analysis. Its predictions $p_{i}$ at all possible sensor locations are computed and stored in a set $M_{0}$. The number of sampled models $N$ depends upon the modeling assumptions and engineer preferences. Thus, there are $N$ sets of predictions $p$ in $M_{0}$.

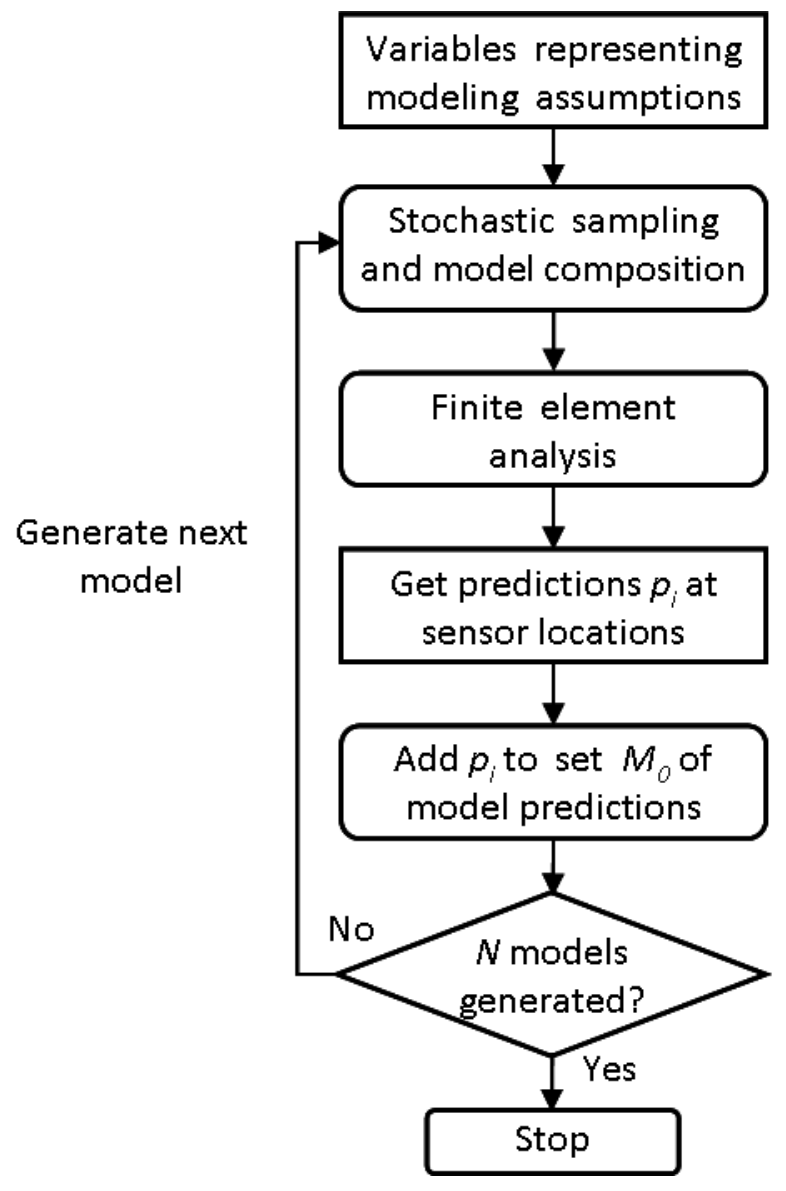

Figure 1: Flowchart explaining the process for model predictions generation used for sensor placement

Given the set of sampled models $M_{0}$, the algorithm for sensor placement shown in Figure 2 is used. The algorithm maintains a collection of model subsets $M$. In the first iteration, $M$ contains only $M_{0}$. During each iteration, the algorithm finds the biggest subset $M_{\max }$ in collection $M$ and chooses a sensor location $i$ that best separates the model predictions in $M_{\max }$. The algorithm finds location $i$ as 
follows. At each possible sensor location, a histogram containing predictions in $M_{\max }$ is built. Each histogram represents the number of models whose predictions lie within each interval (note that the intervals are defined by the accuracy of the measurement devices). To measure the separation between model predictions, Robert-Nicoud et al. (2005b) used the notion of entropy. The expression used to calculate entropy is the Shannon's entropy function (Shannon and Weaver, 1949). Shannon's entropy function is a mathematical representation for the uncertainty in a set. For a random variable $X$, the entropy $H(X)$ is given by the following equation.

$$
H(X)=-\sum_{i=1}^{|X|} p_{i} \cdot \log \left(p_{i}\right)
$$

$p_{i}$ are the probabilities of the $|X|$ different possible values of $X$. For practical purposes, $0 \cdot \log (0)$ is taken to be 0 . Entropy is a measure of disorder in a distribution. The entropy for a given sensor location is calculated for the histogram of model predictions (the histogram being the discretization process). The probability $p_{i}$ of an interval is the ratio of the number $N_{i}$ of models in the interval by the total number of models $N_{\text {tot }}$. Equation 1 comes from the field of information theory. The disorder, and therefore entropy, is at maximum when model predictions show wide dispersion. At the best measurement locations, model predictions should have maximum variation. The algorithm iteratively chooses the locations with highest entropy for sensor placement. The algorithm stops when there is no further improvement to the maximum number of non-identifiable models, i.e., $M_{\max }$ does not become smaller.

\section{Representative Bridge}

A bridge that was designed by Maillart in 1933 (Figure 3) is used to illustrate the sensor configuration methodology. Still standing today, the Schwandbach Bridge is an early example of a deck-stiffened open-spandrel arch and has been named by Billington (1979) "to be one of the two or three most beautiful concrete bridges ever built". The elliptic horizontal ground-plan curve that is supported by a vertical curved thin-walled arch is also an example of daring structural engineering that has inspired engineers for over seventy years.

The possible sensor locations on the bridge are shown in Figure 4 using node identification numbers. Since this is a bridge with a short span of approximately $50 \mathrm{~m}$, the deflections of the bridge are relatively small. Displacement sensors that can measure with an accuracy of up to $1 \mathrm{~mm}$ are found to be insufficient for system identification. However, inclinometers with fairly high accuracy (approximately 1 microradian) are available and this resolution is acceptable for such system identification. In the following section, damage scenarios that determine the location of the inclinometers on the bridge are explained. 


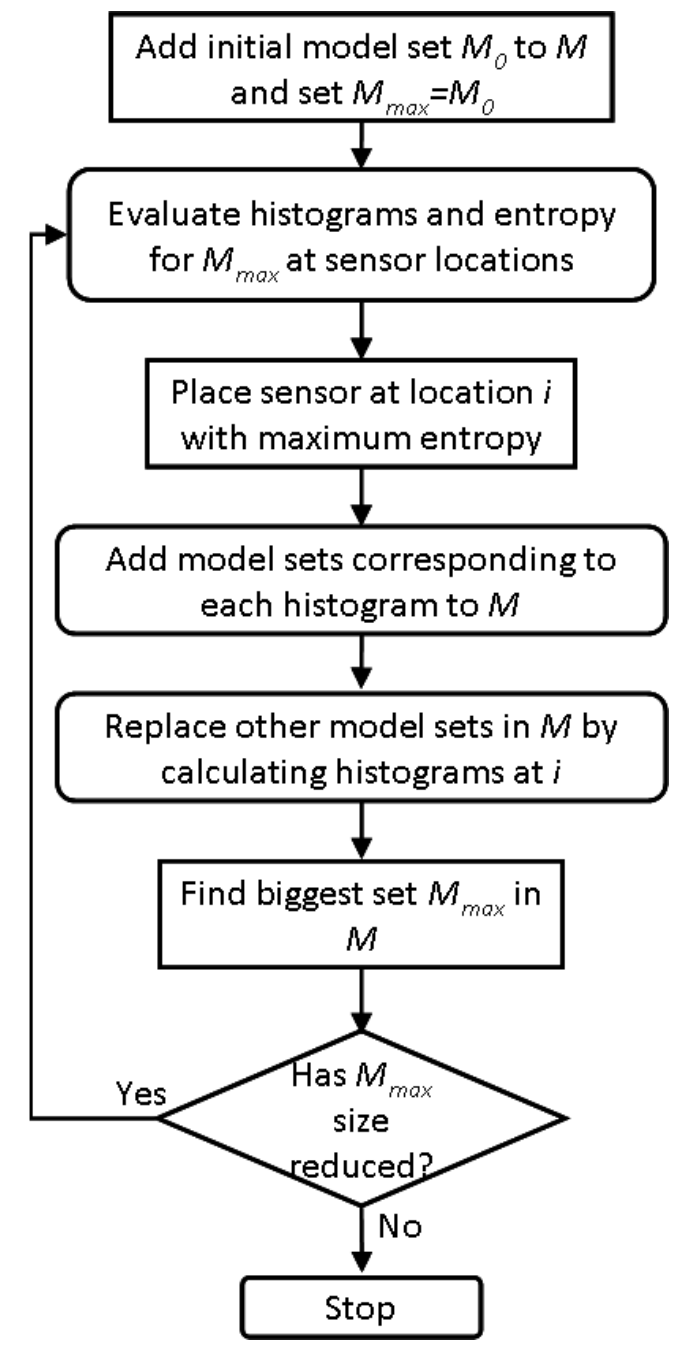

Figure 2: Flowchart of algorithm for sensor placement

\section{Damage scenarios}

Incorporation of damage scenarios in the sensor placement methodology enables selection of measurement locations that maximize the likelihood of identifying future damage. The damage scenarios that are used in this case study are listed in Table 1. The scenarios are derived from a previous experimental study on the Z24 bridge in Switzerland by Maeck et al. (2001). Maeck et al. (2001) created a similar damage in the structure and made measurements under dynamic and static loads. Table 1 also lists the degree of damage to be simulated under each scenario.

In this study, the bridge is modeled as a finite element model in ANSYS. Damage scenarios are simulated by appropriately changing the parameters of the finite element model. Candidate models are generated by stochastic sampling in a model space that consists of all combinations of the damage scenarios listed in 

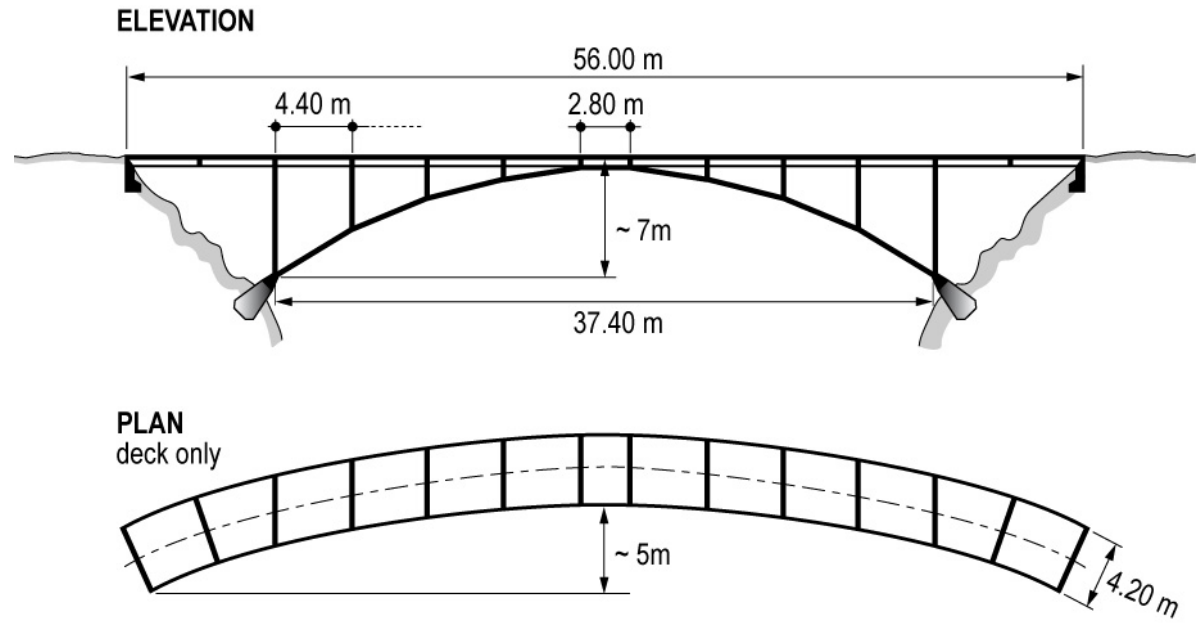

Figure 3: Schema of the Schwandbach Bridge used in the case study

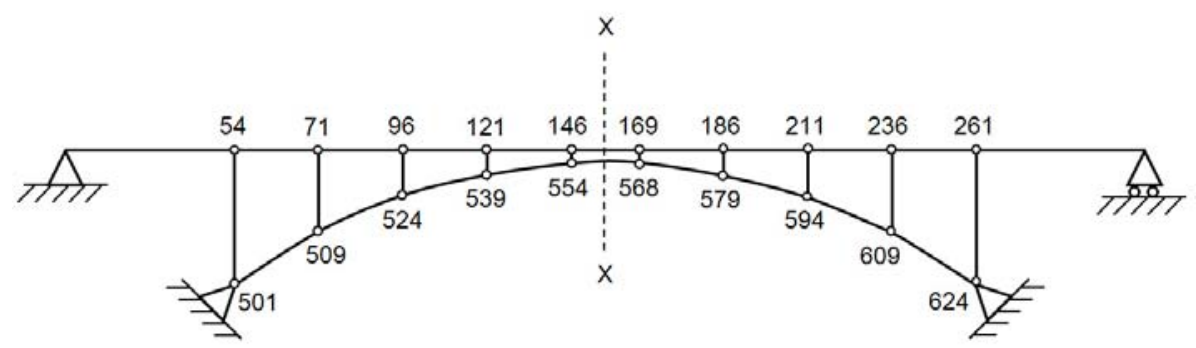

Figure 4: Potential sensor locations on the Schwandbach bridge are shown as the node identification numbers that were used during simulation

Table 1.

\section{Results}

A set containing 5000 damage models of Schwandbach Bridge is created in order to represent the space of possible models. The number of possible sensor locations is 20 (see figure 4 for details). The size of the solution space is $2^{20}$ since a sensor may or may not be present at a given location. This space is sufficiently large to illustrate key aspects of the methodology.

The algorithm described in Section 2 is used for sensor placement. The number of intervals, $I$, depends on the sensor precision and is an integer greater than or equal to 2. For this simulation, $I=10$. Table 2 shows the results obtained. Schwandbach Bridge is nearly symmetrical with respect to a center line (depicted by line $\mathrm{X}-\mathrm{X}$ in Figure 4) and the damage scenarios considered in this study are also symmetrical. Therefore, the sensor placement algorithm explicitly imposes symmetry by placing a pair of sensors in every iteration. During each iteration, 
Table 1: Damage scenarios considered and possible causes

\begin{tabular}{|c|c|c|}
\hline Damage & Causes & Damage limits \\
\hline Arch abutment settlement & $\begin{array}{l}\text { Settlement of subsoil, } \\
\text { erosion }\end{array}$ & $0-30 \mathrm{~cm}$ \\
\hline Arch abutment tilt & $"$ & $0-2^{o}$ \\
\hline Deck support settlement & $"$ & $0-30 \mathrm{~cm}$ \\
\hline Cracks on inner girder & $\begin{array}{l}\text { Overload, settlement of } \\
\text { subsoil, erosion }\end{array}$ & $0-50 \%$ stiffness reduction \\
\hline Cracks on outer girder & $"$ & $"$ \\
\hline Spalling of inner girder & $\begin{array}{l}\text { Frost thawing } \\
\text { cycles, temperature } \\
\text { shock while } \\
\text { applying de-icing salt }\end{array}$ & $"$ \\
\hline Spalling of outer girder & , & $"$ \\
\hline
\end{tabular}

the algorithm identifies the best location for the next sensor and then places sensors at both the best location and its corresponding symmetric location. The deployment that provides maximum identifiability is shown in Figure 5. For every consecutive set of sensors placed, the chosen sensors (node id) and the size of the biggest subset of non identifiable models are given in Table 2 .

Table 2: Number of non identified models and the number of sensors placed on the structure

\begin{tabular}{lccccccccc}
\hline Number of sensors & 2 & 4 & 6 & 8 & 10 & 12 & 14 & 16 & $\ldots$ \\
\hline Node \# & 624 & 236 & 609 & 54 & 568 & 96 & 169 & 539 & \\
Symmetric node \# & 501 & 71 & 509 & 261 & 554 & 211 & 146 & 579 & \\
Non-id. models & 181 & 113 & 86 & 80 & 63 & 54 & 51 & 51 & $\ldots$ \\
\hline
\end{tabular}

After the seventh iteration (fourteen sensors used), there is no decrease in the number of non-identifiable models. This means that placing more than fourteen sensors will not improve the system identification process. Further improvement is only possibly by including new sensor types or adding new sensor locations in the sensor placement strategy. It is understandable that the sensor positions suggested are close to the elements that have been modified to represent damage. The arch abutment settlement and tilt as well the deck support settlement are close to the ends of the structure. The girder elements most susceptible to cracks and spalling were also found to be closer to the ends and have been modeled as such. We find that the sensor positions obtained are closer to the ends, for both the deck/girder as well as the arch. 


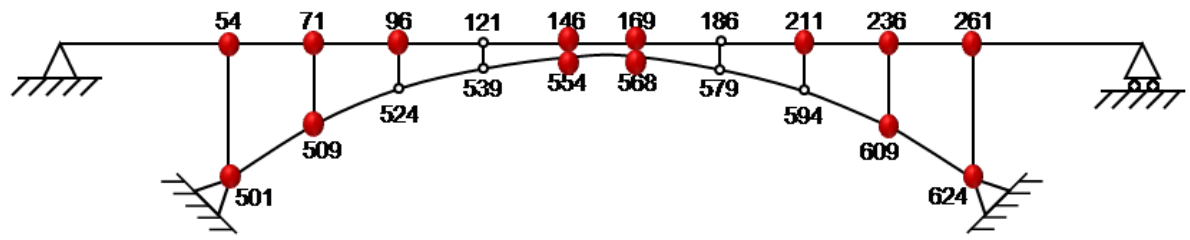

Figure 5: Fourteen sensors deployed on the structure (sensor locations are shown by shaded circles)

\section{Conclusions}

The conclusions of this research are:

- The use of damage scenarios with an entropy-based approach for sensor placement provides a systematic methodology for detecting damage using the least amount of sensors

- For the example studied, starting with 20 potential sensor locations and one sensor type, more than fourteen will not improve damage identification. Further improvement would require other types of sensors or new sensor locations

Future work involves an investigation of the characteristics of sensor configuration with particular attention to the model sets considered during sensor placement. The purpose of placing sensors is to identify potential damage as well as to understand the real behavior of structures in the absence of damage. Therefore, the model prediction sets considered for sensor placement should also include model predictions that represent variations in modeling assumptions. Further studies are needed to determine the proportions of damage scenarios and other behavioral model prediction sets to be used in the sensor placement methodology. A comparison of the sensor placement strategy to a global search based sensor placement method is also underway. Finally, the effect of measurement errors and sensor types with different precisions is also current research.

\section{References}

Akinci, B., Boukamp, F., Gordon, C., Huber, D., Lyons, C., and Park, K. (2006). A formalism for utilization of sensor systems and integrated project models for active construction quality control. Automation in Construction, 15(2):124138.

Berry, J.W., Fleischer, L., Hart, W.E., Phillips, C.A., and Watson, J.-P. (2005). Sensor placement in municipal water networks. Journal of Water Resources Planning and Management, 131(3):237-243. 
Billington, D.P. (1979). Robert Maillart's Bridges. Princeton University Press.

Garrett, J.H., Akinci, B., Matthews, S., Gordon, C., Wang, H., and Sanghvi, V. (2006). Sensor-data driven proactive management of infrastructure systems. In Intelligent Computing in Engineering and Architecture, LNAI 4200, pages 262-284. Springer.

Ljung, L. (1999). System Identification - Theory For the User. Prentice Hall.

Maeck, J., Peeters, B., and de Roeck, G. (2001). Damage identification on the z24 bridge using vibration monitoring. IOP Smart Mater. Struct., 10:512-517.

Robert-Nicoud, Y., Raphael, B., Burdet, O., and Smith, I.F.C. (2005a). Model identification of bridges using measurement data. Computer-Aided Civil and Infrastructure Engineering, 20(2):118-131.

Robert-Nicoud, Y., Raphael, B., and Smith, I.F.C. (2005b). Configuration of measurement systems using shannon's entropy function. Computers and structures, 83(8-9):599-612.

Saitta, S., Raphael, B., and Smith, I.F.C. (2006). Rational design of measurement systems using information science. In Proceedings of IABSE Conference in Budapest, volume IABSE Report 92, page 118:119.

Shannon, C. and Weaver, W. (1949). The Mathematical Theory of Communication. University of Illinois Press.

Smith, I.F.C. (2005). Sensors, models and videotape. In Proceedings of the 2005 ASCE International Conference on Computing in Civil Engineering, Reston VA. American Society of Civil Engineers. CDROM. 